MATHEMATICS OF COMPUTATION

Volume 68, Number 225, January 1999, Pages 1-23

S 0025-5718(99)01025-X

\title{
THE DISCRETE PLATEAU PROBLEM: ALGORITHM AND NUMERICS
}

\author{
GERHARD DZIUK AND JOHN E. HUTCHINSON
}

\begin{abstract}
We solve the problem of finding and justifying an optimal fully discrete finite element procedure for approximating minimal, including unstable, surfaces. In this paper we introduce the general framework and some preliminary estimates, develop the algorithm, and give the numerical results. In a subsequent paper we prove the convergence estimate.

The algorithmic procedure is to find stationary points for the Dirichlet energy within the class of discrete harmonic maps from the discrete unit disc such that the boundary nodes are constrained to lie on a prescribed boundary curve. An integral normalisation condition is imposed, corresponding to the usual three point condition. Optimal convergence results are demonstrated numerically and theoretically for nondegenerate minimal surfaces, and the necessity for nondegeneracy is shown numerically.
\end{abstract}

\section{INTRODUCTION}

A minimal surface or solution of the Plateau Problem is a surface in $\mathbb{R}^{n}$ which has the topology of the unit disc, spans a given boundary curve $\Gamma \subset \mathbb{R}^{n}$, and either minimises, or more generally is stationary for, the area functional. Comprehensive references for the classical theory of minimal surfaces can be found in the books by Dierkes, Hildebrandt, Küster, Wohlrab [DHKW] and by J.C.C. Nitsche [N2]. Various formulations of the problem are discussed in Section 3.

In this and a subsequent paper [DH4] we solve the problem of finding and justifying an optimal, fully discrete, finite element procedure for approximating general (including unstable) minimal surfaces. In [DH1] we developed a boundary integral method but the effects of numerical quadrature were not considered. An outline of some of this work appears in the conference proceedings [DH3], where there is also a summary of the algorithm for the finite element method. Apart from this, the main related results are due to Tsuchiya. He gives an existence proof for discrete minimal surfaces in [T2, T3] and proves convergence to a continuous solution in the $H^{1}(D)$-norm. Although this seems to be the first complete convergence result for the approximation of parametric minimal surfaces, an indirect argument is used which does not give any order of convergence with respect to the grid size, and convergence is proved only for minimisers.

The Plateau Problem is highly nonlinear and the techniques here can be applied to other geometric and nonlinear problems. Technical difficulties also arise since

Received by the editor August 26, 1996.

1991 Mathematics Subject Classification. Primary 65N30; Secondary 49Q05, 53A10.

Key words and phrases. minimal surface, finite elements, order of convergence, Plateau Problem. 
local uniform convexity (more generally, nondegeneracy) of the energy functional is measured with respect to one norm, but the energy functional is only smooth with respect to a stronger norm. Moreover, the discrete energy functional is not the restriction of the original functional (unlike the situation in [DH1]).

Let $D$ be the unit disc in $\mathbb{R}^{2}$. An equivalent characterisation of minimal surface which we will use is the following. Let $\mathcal{F}$ be the class of maps $u: \bar{D} \rightarrow \mathbb{R}^{n}$ such that $\left.u\right|_{\partial D}: \partial D \rightarrow \Gamma$ is monotone, $\left.u\right|_{\partial D}$ satisfies a "three-point condition" (see (9) and in our case (21)), and $u$ is harmonic. The function $u \in \mathcal{F}$ is said to be a minimal surface if $u$ is stationary in $\mathcal{F}$ for the Dirichlet energy $\mathcal{D}(u)=\frac{1}{2} \int_{D}|\nabla u|^{2}$. See the fourth definition in Section 3.1. Such maps $u$ provide a harmonic and conformal parametrisation of the corresponding minimal surface.

Following this characterisation, a first approximation to our numerical method is as follows. Let $D_{h}$ be a quasi-uniform triangulation of $D$ with grid size controlled by $h$. Let $\mathcal{F}_{h}$ be the class of continuous piecewise-linear maps $u_{h}: \bar{D}_{h} \rightarrow \mathbb{R}^{n}$ for which $u_{h}\left(\phi_{j}\right) \in \Gamma$ whenever $\phi_{j}$ is a boundary node of $D_{h}$, which satisfy the threepoint condition and which are discrete harmonic. Note that we do not require "monotonicity" of $\left.u_{h}\right|_{\partial D_{h}}$. The function $u_{h} \in \mathcal{F}_{h}$ is said to be a discrete minimal surface if $u_{h}$ is stationary within $\mathcal{F}_{h}$ for the Dirichlet energy $\mathcal{D}\left(u_{h}\right)=\frac{1}{2} \int_{D_{h}}\left|\nabla u_{h}\right|^{2}$. A member of $\mathcal{F}_{h}$ is determined by its values at the boundary nodes. Thus $\mathcal{F}_{h}$ is a finite dimensional (nonlinear) manifold in some high dimensional Euclidean space. The first and second derivatives of the Dirichlet energy restricted to this manifold can be computed from a knowledge of $\Gamma$. From this, one can compute discrete minimal surfaces.

The main convergence result in [DH4] is that if $u$ is a "nondegenerate", harmonic and conformally parametrised minimal surface spanning $\Gamma$, then there exist discrete minimal surfaces $u_{h}$ such that

$$
\left\|u-u_{h}\right\|_{H^{1}\left(D_{h}\right)} \leq c h
$$

where $c$ depends on $\gamma$ and the nondegeneracy constant for $u$ but is independent of $h$.

We now discuss some of the main ideas in our approach. For both computational and theoretical reasons it is important to move the nonlinearity from the constraint manifold and onto the energy functional. Following [St1] and [St2], fix a smooth parametrisation $\gamma: S^{1} \rightarrow \Gamma$, where $S^{1}$ is the unit circle in $\mathbb{R}^{2}$; it will be convenient to distinguish between $S^{1}$ and the boundary $\partial D$ of $D$. See Figure 1. A map $f: \partial D \rightarrow \Gamma$ can be uniquely written in the form $f=\gamma \circ s$, where $s: \partial D \rightarrow S^{1}$. The set of all maps $s: \partial D \rightarrow S^{1}$ is an affine vector space, since we have a well defined notion of what it means to add to such a map $s$ another map from the (ordinary) vector space of maps $\sigma: \partial D \rightarrow \mathbb{R}$. We will restrict considerations to maps $s$ with winding number one, which satisfy a certain normalisation condition (see (21)) and which have a finite "norm" in one of two possible senses. The corresponding affine Banach spaces will be denoted $\mathcal{H}$ and $\mathcal{T}\left(=\mathcal{H} \cap C^{0}\right)$; see Definitions 3.4 and 3.5. Somewhat informally, members of $\mathcal{H}$ have finite "norm" in the $H^{1 / 2}$ sense and members of $\mathcal{T}$ have finite "norm" in the $H^{1 / 2} \cap C^{0}$ sense. The associated spaces of variations are ordinary Banach spaces and are denoted by $H$ and $T\left(=H \cap C^{0}\right)$, respectively.

Rather than the area functional, we will work with the following energy functional $E$. Let $u=\Phi(\gamma \circ s): \partial D \rightarrow \mathbb{R}^{n}$ be the (unique) harmonic extension of $\gamma \circ s$, 


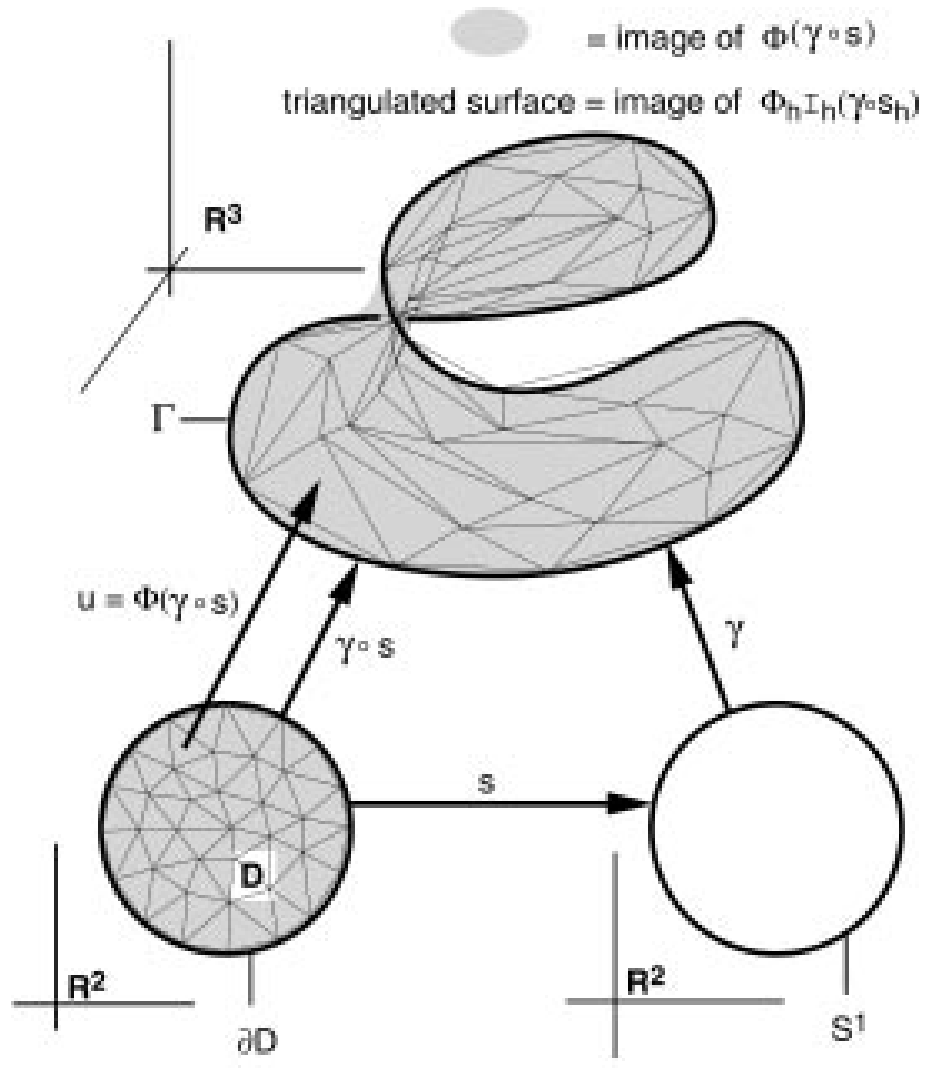

Figure 1

for $s \in \mathcal{T}$. The energy functional $E$ is defined on $\mathcal{T}$ by

$$
E(s)=\frac{1}{2} \int_{D}|\nabla u|^{2}=\frac{1}{2} \int_{D}|\nabla \Phi(\gamma \circ s)|^{2} .
$$

The class of competing maps is now the linear (affine) space $\mathcal{T}$, but the energy functional $E(s)$ is no longer quadratic in $s$. In particular, the Euler equations are highly nonlinear. A parametrised surface $u=\Phi(\gamma \circ s)$ is a minimal surface if $s$ is monotone and stationary for $E$ in $\mathcal{T}$. We remark that $E$ is smooth on $\mathcal{T}$ but not even $C^{1}$ on $\mathcal{H}$, as indicated in one of the remarks preceding Proposition 3.8. On the other hand, "nondegeneracy" is expressed in terms of the $H^{1 / 2}$ norm and so refers to the space $\mathcal{H}$ rather than to $\mathcal{T}$. Members of $\mathcal{H}$ may "just" fail to be continuous and hence to be members of $\mathcal{T}$; counterexamples blow up logarithmically.

Our numerical and theoretical convergence results are obtained under the condition that the minimal surface $u$ be nondegenerate. Such surfaces may have perturbations which strictly decrease area; i.e., the second derivative of the area functional may have negative eigenvalues, but there must be no zero eigenvalues apart from those corresponding to reparametrisatons of the surface. More precisely, the eigenvalues of the second variation $E^{\prime \prime}(s)$ of the energy functional must be bounded away from zero. Minimal surfaces with branch points are always degenerate; a branch point on a minimal surface $u$ is a point at which $\nabla u=0$ - see the examples in 
Section 6 . On the other hand, minimal surfaces without branch points are generically nondegenerate; i.e., arbitrarily small perturbations of the boundary remove any nondegeneracy (see Böhme and Tromba $[\mathrm{BT}]$ ). Moreover, area minimising surfaces in $\mathbb{R}^{3}$ have no branch points, at least in the interior (see Osserman $[\mathrm{O}]$, Alt [A1, A2] and Gulliver [G]). This is not true in $\mathbb{R}^{n}$ if $n \geq 4$. If $\gamma$ is analytic, then area minimisers have no branch points on $\Gamma$; this is not known if $\gamma$ is not analytic.

The integral normalisation condition (21) (analogous to the classical three point condition (9) ) removes a three parameter family of perturbations corresponding to the Möbius group of conformal transformations of the unit disc $D$. This family leaves $E$ invariant and would lead to zero eigenvalues for $E^{\prime \prime}(s)$ at a minimal surface $u=\Phi(\gamma \circ s)$. The integral form is more convenient than the three point condition both numerically and theoretically.

The discrete analogue of $\mathcal{T}$ (and $\mathcal{H}$ ) is the affine subspace $\mathcal{H}_{h}(\subset \mathcal{T} \subset \mathcal{H})$ of continuous maps $s_{h}: \partial D \rightarrow S^{1}$ which are piecewise linear with respect to arc length on the arc segments joining consecutive boundary nodes. Functions $s_{h} \in \mathcal{H}_{h}$ are defined on $\partial D$ rather than on $\partial D_{h}$. However, they may be identified in a one-one manner with maps from the boundary nodes of $D_{h}$ into $S^{1}$, provided the image of each arc segment under $s_{h}$ is less than $\pi$, which is no restriction in practice. We use the $H^{1 / 2}$ norm on $H_{h}$ and the corresponding metric on $\mathcal{H}_{h}$.

Let $u_{h}=\Phi_{h} I_{h}\left(\gamma \circ s_{h}\right)$ be the (unique) discrete harmonic extension of the piecewise linear interpolant $I_{h}\left(\gamma \circ s_{h}\right)$ of $s_{h} \in \mathcal{H}_{h}$. We remark that $I_{h}\left(\gamma \circ s_{h}\right): \partial D_{h} \rightarrow \mathbb{R}^{n}$ is defined on $\partial D_{h}$ and is piecewise linear in the usual Euclidean sense, whereas $\gamma \circ s_{h}$ is defined on $\partial D$. Note also that $I_{h}\left(\gamma \circ s_{h}\right)$ maps the boundary nodes of $\partial D_{h}$ into $\Gamma$, the image of $I_{h}\left(\gamma \circ s_{h}\right)$ is a polygon in $\mathbb{R}^{n}$ and the image of $u_{h}$ is a continuous and piecewise linear surface. The discrete energy functional $E_{h}$ is defined on $\mathcal{H}_{h}$ by

$$
E_{h}\left(s_{h}\right)=\frac{1}{2} \int_{D_{h}}\left|\nabla u_{h}\right|^{2}=\frac{1}{2} \int_{D_{h}}\left|\nabla \Phi_{h} I_{h}\left(\gamma \circ s_{h}\right)\right|^{2} .
$$

Note that whereas $\mathcal{H}_{h} \subset \mathcal{T} \subset \mathcal{H}, E_{h}$ is not the restriction of $E$ to $\mathcal{H}_{h}$. If $s_{h} \in \mathcal{H}_{h}$ is stationary for $E_{h}$, we say $u_{h}=\Phi_{h} I_{h}\left(\gamma \circ s_{h}\right)$ is a discrete minimal surface. Stationary points $s_{h}$ are found by a Newton algorithm which is defined in both abstract and matrix-vector form in Section 5. We note that $E_{h}\left(s_{h}\right)$ depends only on $\gamma$ and the nodal values $s_{h}\left(\phi_{j}\right)$, where $\phi_{j}$ ranges over the boundary nodes of $D_{h}$. The same dependency applies to the first and second derivatives of $E_{h}\left(s_{h}\right)$, which will be explicitly computed.

The first author would like to thank the Centre for Mathematics and its Applications, and the second author would like to thank the Institut für Angewandte Mathematik for their hospitality during the course of this work. Some of the graphics were done with the program GRAPE developed at SFB 256, Bonn and at the University of Freiburg. This research has been partially supported by the Australian Research Council.

\section{Previous numerical methods}

We briefly review previous numerical methods; for more details see [DH1]. 
If the graph of a function $u: \mathbb{R}^{k} \rightarrow \mathbb{R}$ is a ( $k$-dimensional) minimal surface, then it satisfies the minimal surface equation

$$
\sum_{i=1}^{k} D_{i}\left(\frac{D_{i} u}{\sqrt{1+|D u|^{2}}}\right)=0
$$

The problem of numerical approximations in this setting was perhaps first raised by Douglas [Dou2]. He used a finite difference approach, and his paper contains both numerical examples and graphics, but the appropriate techniques to prove convergence were not then available. In the case of a convex domain and $k=2$ there are now optimal estimates in all norms for finite element approximations; see Concus [Co], Johnson \& Thomée [JT], Rannacher [Ra], Ciarlet [Ci].

The most successful numerical approach up to now in the parametric (i.e., nongraphical) setting for polygonal boundary curves uses Courant's function $d(\tau)$, where $\tau=\left\{0<\tau_{1}<\cdots<\tau_{n}<2 \pi\right\}$ varies over partitions of $\partial D$ associated in a natural way to the given polygonal boundary; see [Cou]. This idea was used by Jarausch $[\mathrm{J}]$ to compute approximations to minimal surfaces using finite elements on $D$ which are bilinear with respect to polar coordinates. Drawbacks of the method are that the grid on $D$ varies with $\tau$ and has a singularity at the origin. Jarausch proves convergence of the functional $\mathcal{D}$ with respect to the grid size. Wohlrab [Wo] extended this method to partially free minimal surfaces and to more general variational problems. Heinz proved that with a slight modification in the definition of $d(\tau)$ it becomes an analytic function. The resulting function is called Shiffman's function and was used by Hinze [Hi1, Hi2] to compute minimal surfaces bounded by polygons.

Some numerical work has been done in directly minimising the area functional over various discrete spaces. Of course any such numerical method leads to theoretical and numerical problems because of the invariance of the area functional under arbitrary diffeomorphisms. Wagner [Wa1, Wa2] used the area functional to minimise area for polyhedra spanning a given boundary curve. The same approach was used by Steinmetz [Ste] for more complicated problems involving minimal surfaces, especially partially free minimal surfaces; see also Tsuchiya [T1, Prop. 1]. Parks $[\mathrm{P}]$ approximated minimal surfaces by the level sets of functions of least gradient.

Mean curvature flow was used by Dziuk [Dz] to compute stable minimal surfaces by using finite elements on surfaces; no convergence proof is given. A somewhat similar idea with an infinite time step was used by Pinkall and Polthier [PP] to compute minimal surfaces and their conjugates. A public-domain program "Surface Evolver", which can obtain minimisers for many discrete functionals (including the discrete area functional), has been written by Brakke $[\mathrm{Br}]$. Conditions under which there is a smooth minimal surface near a discrete minimal surface have been obtained by Underwood [U]. Sullivan [Su] has a max flow/min cut type algorithm which uses a polyhedral decomposition of space to obtain approximations to area minimising currents, and he provides a theoretical analysis. In all these cases, in order to obtain reasonable accuracy the numerical estimates require decompositions too fine for current workstations.

Following the lines of the proof of Rado $[\mathrm{R}]$ and Douglas [Dou], Tsuchiya gives an existence proof for discrete minimal surfaces in [T2, T3] and proves convergence to a continuous solution in the $H^{1}(D)$-norm. Wilson [Wi] used the Douglas boundary 
integral form of the Dirichlet functional for harmonic $u$,

$$
\overline{\mathcal{D}}\left(\left.u\right|_{\partial D}\right):=\frac{1}{16 \pi} \int_{\partial D} \int_{\partial D} \frac{|u(\phi)-u(\bar{\phi})|^{2}}{\sin ^{2}\left(\frac{\phi-\bar{\phi}}{2}\right)} d \phi d \bar{\phi}=\mathcal{D}(u),
$$

in order to compute minimal surfaces. Hutchinson $[\mathrm{Hu}]$ minimised the conformal energy $\mathcal{D}(u)-\mathcal{A}(u)$, which is always $\geq 0$ and equals 0 if and only if $u$ is a minimal surface. In some situations this has significant numerical advantages over minimising the Dirichlet energy. In addition, arbitrary and not necessarily stable minimal surfaces can be found by a minimisation procedure.

In [DH1] (see also [DH3]) we obtained discrete minimal surfaces by, to be somewhat imprecise, computing stationary points of a boundary integral restricted to certain finite element spaces. More precisely, let $\gamma: S^{1} \rightarrow \Gamma$ be a fixed parametrisation of the given boundary curve. For $h>0$ let $\mathcal{G}_{h}$ be a partition of $\partial D$ such that the distance between successive nodes is bounded above and below by multiples of $h$ which are independent of $h$. Let $\mathcal{H}_{h}$ be the set of maps $s_{h}: \partial D \rightarrow S^{1}$ which are continuous and piecewise linear with respect to arc length along arc segments in $\mathcal{G}_{h}$. Let

$$
E\left(s_{h}\right)=\mathcal{D}\left(u_{h}\right)=\overline{\mathcal{D}}\left(\gamma \circ s_{h}\right),
$$

where $u_{h}$ is the harmonic extension of $\gamma \circ s$. Note that $u_{h}$ is smooth, not discrete, in the interior of $D$. We say that $u_{h}$ is a semi-discrete minimal surface if $s_{h}$ is stationary for $E$ in $\mathcal{H}_{h}$. We showed that if $u$ is a minimal surface, then there is a sequence of semi-discrete minimal surfaces $u_{h}$ such that

$$
\left\|u-u_{h}\right\|_{H^{1}(D)} \leq c h^{3 / 2},
$$

and in [DH2] we proved an $O\left(h^{5 / 2}\right)$ estimate for the $L^{2}$ norm. Although this gives a better order of convergence than the current method, the algorithm is computationally much more intensive. In addition, as noted before, the theoretical analysis in [DH1] ignores the effect of quadrature approximations in computing the boundary integral $\overline{\mathcal{D}}\left(\gamma \circ s_{h}\right)$.

\section{The smooth Plateau Problem}

3.1. Theoretical background. The classical Plateau Problem consists of finding a least area surface of disk type spanning a given wire in $\mathbb{R}^{n}$. The wire is represented by a curve $\Gamma$ homotopic to a circle, i.e., by a Jordan curve.

Let

$$
D=\{z=(x, y)|| z \mid<1\}
$$

denote the unit disk in $\mathbb{R}^{2}$. One then looks for functions

$$
u: \bar{D} \rightarrow \mathbb{R}^{n}
$$

such that $\partial D$ is mapped onto $\Gamma$ in a one-one (i.e., monotone) way and such that $u$ is a minimum for the area functional

$$
\mathcal{A}(u)=\int_{D}\left|u_{x} \times u_{y}\right| .
$$

To be more precise, one is interested in finding $u$ in the set of admissable functions

$$
\mathcal{C}^{\prime}(\Gamma)=\left\{v \in H^{1}(D)^{n} \cap C^{0}(\partial D)^{n}|v|_{\partial D}: \partial D \rightarrow \Gamma \text { is monotone }\right\}
$$


such that

$$
\mathcal{A}(u)=\inf _{v \in \mathcal{C}^{\prime}(\Gamma)} \mathcal{A}(v)
$$

More generally, one defines $u \in \mathcal{C}^{\prime}(\Gamma)$ to be a minimal surface (or solution of the Plateau Problem) if $u$ is stationary for $\mathcal{A}$. A minimal surface need not be a minimiser, or even stable.

The area functional $\mathcal{A}$ equals Dirichlet's integral

$$
\mathcal{D}(u)=\frac{1}{2} \int_{D}\left|u_{x}\right|^{2}+\left|u_{y}\right|^{2}
$$

if and only if $u$ is conformally parametrized; see (12). In general we have $\mathcal{A}(u) \leq$ $\mathcal{D}(u)$. Since $\mathcal{A}$ is invariant under arbitrary diffeomorphisms it is easier to work with $\mathcal{D}$. A variational problem essentially equivalent to (6) is to solve

$$
\mathcal{D}(u)=\inf _{v \in \mathcal{C}^{\prime}(\Gamma)} \mathcal{D}(v)
$$

Dirichlet's integral is conformally invariant, i.e., $\mathcal{D}(u)=\mathcal{D}(u \circ w)$ for any conformal diffeomorphism (Möbius transformation) $w$ of the disc $D$. Thus one should factor out the conformal group by some additional assumption on the class $\mathcal{C}^{\prime}(\Gamma)$ of admissable functions. Classically this is done by imposing a three-point condition on $\left.u\right|_{\partial D}$. Choose any three distinct points $z_{i}$ on $\partial D$ and three distinct points $P_{i}$ on $\Gamma$ and impose the condition

$$
u\left(z_{i}\right)=P_{i}
$$

for $i=1,2,3$. Consequently the class of admissable functions is changed to

$$
\begin{gathered}
\mathcal{C}(\Gamma)=\left\{u \in H^{1}(D)^{n} \cap C^{0}(\partial D)^{n}|u|_{\partial D}: \partial D \rightarrow \Gamma\right. \text { monotone } \\
\text { and } u \text { satisfies }(9) .\}
\end{gathered}
$$

Such a three-point condition is numerically unpleasant because it is an $L^{\infty}$-condition. The classical three-point condition will be replaced by an $L^{2}$-condition for our purposes; see Definition 3.4. An essentially equivalent second definition then is that $u \in \mathcal{C}(\Gamma)$ is a minimal surface if $u$ is stationary for $\mathcal{D}$.

A function $u$ is stationary for $\mathcal{D}$ iff $u$ is harmonic and conformal. Thus an equivalent third definition is that $u \in \mathcal{C}(\Gamma)$ is a minimal surface if

$$
\begin{gathered}
\Delta u=0, \\
\left|u_{x}\right|=\left|u_{y}\right|, u_{x} \cdot u_{y}=0 .
\end{gathered}
$$

This definition shows the highly nonlinear character of the Plateau Problem not only because of the conformality relations (12) but also because of the definiton of the boundary condition in the class $\mathcal{C}(\Gamma)$. Note that $u$ has a free boundary on the one dimensional manifold $\Gamma$.

Finally, an equivalent fourth definition is that $u$ is a minimal surface if $u \in$ $\mathcal{C}(\Gamma) \cap\{v \mid \Delta v=0\}$ and $u$ is stationary in this class for $\mathcal{D}$. An advantage of this definition is that harmonic maps are uniquely determined by their boundary values, and so we are essentially looking for stationary points of a certain functional defined over a class of boundary maps from $\partial D$ to $\Gamma$. This is the approach we will pursue, but with a modification to the three point condition.

The following theorem of Douglas [Dou] and Rado [R] states that the Plateau Problem has at least one solution. 
Theorem 3.1. Let $\Gamma$ be a rectifiable Jordan curve in $\mathbb{R}^{3}$. Then the problem

$$
\mathcal{D}(u)=\inf _{v \in \mathcal{C}(\Gamma)} \mathcal{D}(v)
$$

has a solution $u \in \mathcal{C}(\Gamma)$; moreover $u$ is harmonic and conformal. Also, $u$ solves the problem

$$
\mathcal{A}(u)=\inf _{v \in \mathcal{C}(\Gamma)} \mathcal{A}(v)
$$

The boundary map $\left.u\right|_{\partial D}$ is a topological mapping onto the curve $\Gamma$.

Apart from the Douglas-Rado solutions, many curves $\Gamma$ have other, possibly unstable, solutions to the Plateau Problem. In particular, see the examples in Section 6. It is an open question whether there exist at most finitely many solutions of the Plateau Problem for a given curve $\Gamma$. From the work of Böhme and Tromba $[\mathrm{BT}]$ it is known that generically the number of solutions is finite. A result of Tomi [To] guarantees that there are only finitely many absolute minimisers for an analytic boundary curve $\Gamma$.

Asymptotic convergence of the numerical method requires regularity of solutions up to the boundary. This was proved by Hildebrandt [Hil], Nitsche [N1], Jäger [Ja] and Heinz [He].

Theorem 3.2. Let $u$ be a minimal surface which maps an open arc $A \subset \partial D$ into an open portion $\Gamma^{\prime} \subset \Gamma$ and assume that $\Gamma^{\prime} \in C^{k, \alpha}$ for some $k \in \mathbb{N}$ and some $0<\alpha<1$. Then $u \in C^{k, \alpha}(D \cup A)$.

3.2. Reformulation of the Problem. Assume that $\Gamma$ is a Jordan curve in $\mathbb{R}^{n}$ with regular $C^{r}$-parametrisation

$$
\gamma: S^{1} \rightarrow \Gamma
$$

where $r \geq 3$. Although $\partial D=\left\{z \in \mathbb{R}^{2}|| z \mid=1\right\}$ and $S^{1}=\left\{e^{i \phi} \mid 0 \leq \phi<\right.$ $2 \pi\} \cong \mathbb{R} / 2 \pi \cong[0,2 \pi)$ are naturally isomorphic, we will consider $S^{1}$ as the domain of the fixed parametrisation $\gamma$ of $\Gamma$, and consider $\partial D$ as the boundary of the fixed parameter domain for various parametrised surfaces.

For $f: \partial D \rightarrow \mathbb{R}^{n}$ we denote by

$$
\Phi(f): \bar{D} \rightarrow \mathbb{R}^{n}
$$

its unique harmonic extension to $D$ specified by

$$
\begin{aligned}
\Delta \Phi(f) & =0 \text { in } D, \\
\Phi(f) & =f \text { on } \partial D .
\end{aligned}
$$

For $f: \partial D \rightarrow \mathbb{R}$ the $H^{1 / 2}(\partial D)$ seminorm is defined by

$$
|f|_{H^{1 / 2}(\partial D)}^{2}=\int_{\partial D} \int_{\partial D} \frac{|f(\phi)-f(\bar{\phi})|^{2}}{|\phi-\bar{\phi}|^{2}} d \phi d \bar{\phi},
$$

the corresponding norm is given by

$$
\|f\|_{H^{1 / 2}}^{2}=\|f\|_{L^{2}}^{2}+|f|_{H^{1 / 2}}^{2}
$$

and the associated inner product is denoted by $(,)_{H^{1 / 2}}$. It is standard that

$$
\Phi: H^{1 / 2}\left(\partial D, \mathbb{R}^{n}\right) \rightarrow H^{1}\left(D, \mathbb{R}^{n}\right)
$$

is a bounded linear map with bounded inverse. 
For future reference we note the following properties which follow from (17).

$$
\begin{aligned}
\|f g\|_{H^{1 / 2}} & \leq c\|f\|_{C^{1}}\|g\|_{H^{1 / 2}}, \\
\|g \circ s\|_{H^{1 / 2}} & \leq c\|g\|_{C^{1}}\|s\|_{H^{1 / 2}} .
\end{aligned}
$$

Now we can define the energy functional $E$ which replaces Dirichlet's integral in (13) and which is defined on certain maps $s: \partial D \rightarrow S^{1}$.

Definition 3.3. For $s: \partial D \rightarrow S^{1}$ such that $\gamma \circ s \in H^{1 / 2}\left(\partial D, \mathbb{R}^{n}\right)$, let

$$
E(s)=\frac{1}{2} \int_{D}|\nabla \Phi(\gamma \circ s)|^{2}=\mathcal{D}(\Phi(\gamma \circ s)) .
$$

That is, $E(s)=\mathcal{D}(u)$ where $u$ is the harmonic extension $\Phi(\gamma \circ s)$ of $\gamma \circ s$. Notice that $E(s)$ is comparable to $|\gamma \circ s|_{H^{1 / 2}}^{2}$. As domain for the functional $E$ one first chooses a suitable space $\mathcal{H}$; see Definition 3.4. Loosely speaking, $\mathcal{H}$ consists of those $H^{1 / 2}$ maps $s: \partial D \rightarrow S^{1}$ which wind once around $S^{1}$ and satisfy a certain normalisation condition; see (21). However, in order to obtain a differentiable functional, it will be necessary to restrict $E$ to the subspace $\mathcal{T}$ of continuous members of $\mathcal{H}$.

In Definition 3.4 and elsewhere, points $e^{i \phi}=(\cos \phi, \sin \phi) \in \partial D\left(\right.$ or $\left.\in S^{1}\right)$ are usually, but not always, denoted by the corresponding angle $\phi$. Note that $\phi$ is well defined by $e^{i \phi}$ only up to multiples of $2 \pi$.

Definition 3.4. The Hilbert space $H$ is defined by

$$
H=\left\{\xi:\left.\partial D \rightarrow \operatorname{Re}|| \xi\right|_{H^{1 / 2}}<\infty \text { and (21) is satisfied }\right\},
$$

where

$$
\int_{0}^{2 \pi} \xi(\phi) d \phi=0, \quad \int_{0}^{2 \pi} \xi(\phi) \cos \phi d \phi=0, \quad \int_{0}^{2 \pi} \xi(\phi) \sin \phi d \phi=0 .
$$

The norm on $H$ is $\|\cdot\|_{H^{1 / 2}}$, which by the first condition in (21) and Poincaré's inequality is equivalent to $|\cdot|_{H^{1 / 2}}$. The corresponding affine Hilbert space $\mathcal{H}$ is the space of maps $s: \partial D \rightarrow S^{1}$ such that

$$
s(\phi)=\phi+\sigma(\phi)
$$

for some $\sigma \in H$. Note that addition in (22) is well defined.

Definition 3.5. The Banach space $T$ is defined by

$$
T=H \cap C^{0}(\partial D, \mathbb{R})
$$

with norm

$$
\|\xi\|_{T}=\|\xi\|_{H^{1 / 2}}+\|\xi\|_{C^{0}} .
$$

The corresponding affine space $\mathcal{T}$ is defined by

$$
\mathcal{T}=\mathcal{H} \bigcap C^{0}\left(\partial D, S^{1}\right) .
$$

Because $s \in \mathcal{H}$ is a periodic perturbation of the "identity" map (see (22)), we say $s$ winds once around $S^{1}$. Notice that the function $\sigma$ in (22) is well defined by $s$ up to integer multiples of $2 \pi$ at each point. But one can show that the only $H^{1 / 2}$ functions whose values lie in the set $\{2 k \pi \mid k$ an integer $\}$ are integer multiples of the constant function $2 \pi$. It then follows from the first condition in (21) that $\sigma$ is in fact uniquely determined by $s$. The space of variations at $s \in \mathcal{H}$ or $s \in \mathcal{T}$ is then naturally identified with $H$ or $T$, respectively. 
The normalisation conditions (21) are the analogue of the three-point condition (9). More precisely, it follows from the conformal invariance of Dirichlet's integral that $E(s)=E(s \circ g)$ for any $g: \partial D \rightarrow \partial D$, which is the restriction of a Möbius transformation. However, for any continuous monotone $\bar{s}: \partial D \rightarrow \partial D$ there exists a (unique) $s=\bar{s} \circ g$, where $g$ is the restriction of a Möbius transformation, such that $s$ satisfies (21) (see the Appendix to Section 3 in [DH0] for a proof). Thus there is no loss of generality in working in the class of $s$ satisfying (21).

The energy functional $E$ is well defined and finite on $\mathcal{H}$ (see Proposition 3.7) and conversely, if $s$ is continuous and $E(\gamma \circ s)$ is finite, then $s \in \mathcal{H}$; see [St2, II.2.7] and the estimates in [St2, Lemma II.2.6]. Differentiability of $E$ on $\mathcal{T}$ follows from Proposition 3.8 .

We are now ready to give the formulation of the Plateau Problem which we use in this paper.

Definition 3.6. The harmonic function

$$
u=\Phi(\gamma \circ s)
$$

is a minimal surface spanning $\Gamma$, or a solution of the Plateau Problem for $\Gamma$, if and only if $s \in \mathcal{T}$ is monotone and stationary for $E$, i.e.,

$$
\left\langle E^{\prime}(s), \xi\right\rangle=0, \quad \forall \xi \in T .
$$

The equivalence of this with the formulations of Plateau's Problem in Section 3.1 is established in [St2]; the main point being to use the stationarity condition to first establish the regularity result $\Phi(\gamma \circ s) \in H^{2}\left(D, \mathbb{R}^{n}\right)$. Finding stationary points for $E$ is thus equivalent to solving the nonlinear system (11) and (12).

3.3. Estimates for the energy functional. Although $\mathcal{H}$ and $\mathcal{T}$ are only affine Banach spaces, it will be convenient to introduce the notation

$$
\|s\|=1+\|\sigma\|
$$

where $s(\phi)=\phi+\sigma(\phi)$, for various norms on $\sigma$. Note that $\|s\| \geq 1$; we will use this frequently.

We now have:

Proposition 3.7. $E: \mathcal{H} \rightarrow \mathbb{R}$ and

$$
E(s) \leq c\|\gamma\|_{C^{1}}^{2}\|s\|_{H^{1 / 2}}^{2} .
$$

Proof.

$$
E(s)=\frac{1}{2} \int_{D}|\nabla \Phi(\gamma \circ s)|^{2} \leq c|\Phi(\gamma \circ s)|_{H^{1}(D)}^{2} \leq c|\gamma \circ s|_{H^{1 / 2}(\partial D)}^{2} \leq c\|\gamma\|_{C^{1}}^{2}\|s\|_{H^{1 / 2}}^{2},
$$

from (19).

From (20) and formal computation we have

$$
\begin{aligned}
E(s) & =\frac{1}{2} \int_{D}|\nabla u|^{2} \\
\left\langle E^{\prime}(s), \xi\right\rangle & =\left.\frac{d}{d t}\right|_{t=0} E(s+t \xi)=\int_{D} \nabla u \nabla v \\
E^{\prime \prime}(s)(\xi, \xi) & =\left.\frac{d^{2}}{d t^{2}}\right|_{t=0} E(s+t \xi)=\int_{D} \nabla u \nabla w+\int_{D}|\nabla v|^{2},
\end{aligned}
$$


where

$$
u=\Phi(\gamma \circ s), \quad v=\Phi\left(\gamma^{\prime} \circ s \xi\right), \quad w=\Phi\left(\gamma^{\prime \prime} \circ s \xi^{2}\right)
$$

Bilinearity gives a corresponding expression for distinct variations in (26). Note that the evaluation of the derivatives of $E$ requires the solution of Laplace's equation with various boundary conditions. The map $\gamma^{\prime} \circ s \xi$ can be thought of as a tangential vector field defined either along $\partial D$ or along $\Gamma$; the map $v$ is its harmonic extension and can be thought of as the harmonic variation induced by $\xi$.

Under appropriate smoothness assumptions on $\gamma$ it is not difficult to prove that $E$ is a smooth mapping on $\mathcal{T} \subset \mathcal{H}$, see Proposition 3.8. The estimates in Proposition 3.8 cannot be improved by replacing $\|\xi\|_{T}$ by $\|\xi\|_{H^{1 / 2}}$, unless the regularity on $s$ is increased. The main point is that the composition operator $s \mapsto \gamma \circ s$ is smooth on $\mathcal{T}$ but not even $C^{1}$ on $\mathcal{H}$. Although we do not need Proposition 3.8 we include it for comparison with Proposition 3.9. The latter shows that if $s$ is sufficiently smooth, then the linear and bilinear operators $E^{\prime}(s)$ and $E^{\prime \prime}(s)$ defined on $T$ can be extended to bounded linear and bilinear operators defined on $H$. The arguments used in the proof of Proposition 3.9 are simple prototypes of those used in proving the convergence result in [DH4]. The proof of Proposition 3.8 is similar; see [St2] and also Propositions 5.1, 5.2 and 5.3 in [DH1].

Proposition 3.8. If $\gamma \in C^{3}$, then $E \in C^{2}(\mathcal{T}, \mathbb{R})$. Moreover,

$$
\begin{aligned}
\left|\left\langle E^{\prime}(s), \xi\right\rangle\right| & \leq c\|\gamma\|_{C^{2}}^{2}\|s\|_{H^{1 / 2}}^{2}\|\xi\|_{T}, \\
\left|E^{\prime \prime}(s)(\xi, \eta)\right| & \leq c\|\gamma\|_{C^{3}}^{2}\|s\|_{H^{1 / 2}}^{2}\|\xi\|_{T}\|\eta\|_{T} .
\end{aligned}
$$

Proposition 3.9. If $\gamma \in C^{2}$ and $s \in C^{1}$, then

$$
\begin{aligned}
\left|\left\langle E^{\prime}(s), \xi\right\rangle\right| & \leq c\|\gamma\|_{C^{2}}^{2}\|s\|_{C^{1}}^{2}\|\xi\|_{H^{1 / 2}} \\
\left|E^{\prime \prime}(s)(\xi, \eta)\right| & \leq c\|\gamma\|_{C^{2}}^{2}\|s\|_{C^{1}}^{2}\|\xi\|_{H^{1 / 2}}\|\eta\|_{H^{1 / 2}}
\end{aligned}
$$

Proof. From (27) and (25),

$$
\left|\left\langle E^{\prime}(s), \xi\right\rangle\right| \leq|u|_{H^{1}(D)}|v|_{H^{1}(D)}=I_{1} I_{2} .
$$

But

$$
I_{1} \leq c|\gamma \circ s|_{H^{1 / 2}(\partial D)} \leq c\|\gamma\|_{C^{1}}\|s\|_{C^{1}} \quad \text { by }(19)
$$

and

$$
I_{2} \leq c\left|\gamma^{\prime} \circ s \xi\right|_{H^{1 / 2}(\partial D)} \leq c\left\|\gamma^{\prime} \circ s\right\|_{C^{1}}\|\xi\|_{H^{1 / 2}} \leq c\|\gamma\|_{C^{2}}\|s\|_{C^{1}}\|\xi\|_{H^{1 / 2}}
$$

by (18) and (19).

For the second derivative we may assume $\eta=\xi$. Then from (26)

$$
E^{\prime \prime}(s)(\xi, \xi)=\int_{D}|\nabla v|^{2}+\int_{D} \nabla u \nabla w=\int_{D}|\nabla v|^{2}+\int_{\partial D} \frac{\partial u}{\partial \nu} w=I_{1}+I_{2},
$$

since $u$ is harmonic. But

$$
I_{1}=|v|_{H^{1}(D)}^{2} \leq c\left|\gamma^{\prime} \circ s \xi\right|_{H^{1 / 2}}^{2} \leq c\left\|\gamma^{\prime} \circ s\right\|_{C^{1}}^{2}\|\xi\|_{H^{1 / 2}}^{2} \leq c\|\gamma\|_{C^{2}}^{2}\|s\|_{C^{1}}^{2}\|\xi\|_{H^{1 / 2}}^{2}
$$


from (27), (18) and (19). Also

$$
\begin{aligned}
I_{2} & \leq\left\|\frac{\partial u}{\partial \nu}\right\|_{L^{2}(\partial D)}\|w\|_{L^{2}(\partial D)} \\
& \leq|u|_{H^{1}(\partial D)}\|w\|_{L^{2}(\partial D)} \quad \text { since } \int_{\partial D}\left|\frac{\partial u}{\partial \nu}\right|^{2}=\int_{\partial D}\left|\frac{\partial u}{\partial \tau}\right|^{2} \text { where } \tau \text { is the } \\
& \quad \text { unit tangent vector, using a Fourier series expansion } \\
& \leq c|\gamma \circ s|_{H^{1}}\left\|\gamma^{\prime \prime} \circ s \xi^{2}\right\|_{L^{2}} \quad \text { from }(27) \\
& \leq c\|\gamma\|_{C^{1}}\|s\|_{C^{1}}\|\gamma\|_{C^{2}}\|\xi\|_{L^{4}}^{2} \\
& \leq c\|\gamma\|_{C^{2}}^{2}\|s\|_{C^{1}}\|\xi\|_{H^{1 / 2}}^{2} \quad \text { by a Sobolev embedding theorem. }
\end{aligned}
$$

This gives the estimate for $E^{\prime \prime}$.

3.4. Nondegeneracy for the energy functional. We will need to consider the second order behaviour of $E$ near a stationary point $s \in \mathcal{T}$. More generally, if $s \in \mathcal{H} \cap C^{1}$ and $\gamma \in C^{2}$ (in particular, if $s$ is stationary and $\gamma \in C^{2}$ by regularity theory; see [DH4]), we use Proposition 3.9 to define the bounded self-adjoint map

$$
\nabla^{2} E(s): H \rightarrow H
$$

by

$$
\left(\nabla^{2} E(s)(\xi), \eta\right)_{H^{1 / 2}}=E^{\prime \prime}(s)(\xi, \eta)
$$

for all $\xi, \eta \in H$. Write

$$
\begin{aligned}
H & =H^{-} \oplus H^{0} \oplus H^{+} \\
\xi & =\xi^{-}+\xi^{0}+\xi^{+} \quad \text { if } \xi \in H,
\end{aligned}
$$

for the orthogonal decomposition generated by the eigenfunctions of $\nabla^{2} E(s)$ having negative, zero, and positive eigenvalues, respectively. It is not difficult to show from elliptic theory that $H^{-}$and $H^{0}$ are finite dimensional and that their members are smooth, depending on the regularity of $\gamma$; see the proof of [St2, Proposition 5.6] and also [DH4].

If $s$ is monotone and stationary for $E$, we say

$$
s \text { is nondegenerate if } H^{0}=\{0\} .
$$

The corresponding minimal surface $u=\Phi(\gamma \circ s)$ is also said to be nondegenerate; see [BT]. If $s$ is nondegenerate it follows from standard arguments (see [DH1, Proposition 4.9(iii)]) that the eigenvalues of $\nabla^{2} E(s)$ are bounded away from zero. In this case we define

$$
\begin{aligned}
\lambda^{+} & =\inf \left\{\mu \mid \mu \text { is a positive eigenvalue of } \nabla^{2} E(s)\right\}>0 \\
\lambda^{-} & =\inf \left\{-\mu \mid \mu \text { is a negative eigenvalue of } \nabla^{2} E(s)\right\}>0 \\
\lambda & =\min \left\{\lambda^{+}, \lambda^{-}\right\}>0 .
\end{aligned}
$$

In particular, if $\xi \in H$,

$$
E^{\prime \prime}(s)\left(\xi, \xi^{+}-\xi^{-}\right)=E^{\prime \prime}(s)\left(\xi^{+}, \xi^{+}\right)-E^{\prime \prime}(s)\left(\xi^{-}, \xi^{-}\right) \geq \lambda\|\xi\|_{H^{1 / 2}}^{2} .
$$

We call $\lambda$ the nondegeneracy constant for $s$. 


\section{The discrete Plateau Problem}

4.1. Discrete function spaces. Let $\mathcal{G}_{h}$ be a quasi-uniform triangulation of $D$ controlled by $h$; i.e., each triangle has diameter at most $h$ and at least $\sigma h$ for some $\sigma>0$ independent of $h$, and has angles bounded away from zero independently of $h$.

Define

$$
\begin{aligned}
D_{h}= & \bigcup\left\{G \mid G \in \mathcal{G}_{h}\right\} \\
\partial D_{h}= & \bigcup\left\{E_{j} \mid 1 \leq j \leq M\right\} \quad \text { where the } E_{j} \text { are the boundary edges, } \\
\mathcal{B}_{h}= & \left\{\phi_{1}, \ldots, \phi_{M}\right\} \quad \text { is the set of boundary nodes, } \\
\mathcal{N}_{h}= & \left\{v_{1}, \ldots, v_{N}\right\} \quad \text { is the set of all nodes, } \\
& \text { where } v_{j}=\phi_{j}=e^{i \phi_{j}} \text { for } j=1, \ldots, M .
\end{aligned}
$$

Suppose $f \in C^{0}\left(\partial D, \mathbb{R}^{n}\right)$. Then the continuous and piecewise linear interpolant $I_{h} f$ is defined on $\partial D_{h}$ (not on $\partial D$ ) by

$$
I_{h} f\left((1-t) e^{i \phi_{j}}+t e^{i \phi_{j+1}}\right)=(1-t) f\left(e^{i \phi_{j}}\right)+t f\left(e^{i \phi_{j+1}}\right)
$$

for $0 \leq t \leq 1,1 \leq j \leq M$. Here and elsewhere, $\phi_{M+1}=\phi_{1}$. Note that the image of $I_{h}(\gamma \circ s)$ is a polygonal approximation to $\Gamma$ defined on $\partial D_{h}$, and $I_{h}(\gamma \circ s)\left(\phi_{j}\right)=$ $\gamma \circ s\left(\phi_{j}\right) \in \Gamma$ for $\phi_{j} \in \mathcal{B}_{h}$. We also need a continuous and piecewise linear (with respect to arc length) interpolant $I_{h}^{\partial D} f$, defined on $\partial D$ (as opposed to $\partial D_{h}$ ), given by

$$
I_{h}^{\partial D} f\left(e^{i\left((1-t) \phi_{j}+t \phi_{j+1}\right)}\right)=(1-t) f\left(e^{i \phi_{j}}\right)+t f\left(e^{i \phi_{j+1}}\right)
$$

for $0 \leq t \leq 1,1 \leq j \leq M$.

In order to compare functions defined on $\partial D$ with functions defined on $\partial D_{h}$, define the projection $\pi: \partial D \rightarrow \partial D_{h}$ by

$$
\pi\left(e^{i\left((1-t) \phi_{j}+t \phi_{j+1}\right)}\right)=(1-t) e^{i \phi_{j}}+t e^{i \phi_{j+1}}
$$

for $0 \leq t \leq 1,1 \leq j \leq M$. Thus $\pi$ maps the small arc on $\partial D$ joining two adjacent boundary nodes to the line segment on $\partial D_{h}$ joining the same two nodes; the map is linear with respect to arc length on the arc and with respect to ordinary length on the line segment. Finally, note that

$$
I_{h}^{\partial D} f=I_{h} f \circ \pi .
$$

As noted before, instead of working directly with maps $f: \partial D \rightarrow \Gamma$, we work with the corresponding maps $s: \partial D \rightarrow S^{1}$, where $f=\gamma \circ s$. For this purpose we consider the following discrete spaces.

\section{Definition 4.1.}

$$
\begin{aligned}
& H_{h}=\left\{\xi_{h} \in C^{0}(\partial D, \mathbb{R}) \mid \xi_{h} \in P_{1}\left(\pi^{-1}\left[E_{j}\right]\right) \forall j, \xi_{h} \text { satisfies }(21)\right\} \\
& \mathcal{H}_{h}=\left\{s_{h} \in C^{0}\left(\partial D, S^{1}\right) \mid s_{h}(\phi)=\phi+\sigma_{h}(\phi) \text { for some } \sigma_{h} \in H_{h}\right\}
\end{aligned}
$$

By $P_{1}\left(\pi^{-1}\left[E_{j}\right]\right)$ we mean the set of polynomials of degree one over the arc $\pi^{-1}\left[E_{j}\right]$.

Thus $H_{h} \subset T \subset H$ and $H_{h}$ is an $M-3$ dimensional vector space, where "3" comes from the number of constraints in (21). Notice that these constraints correspond to linear equations for the $\xi_{h}\left(\phi_{j}\right)$. Moreover, $\mathcal{H}_{h} \subset \mathcal{T} \subset \mathcal{H}, \mathcal{H}_{h}$ is an affine space of 
dimension $M-3$, and the space of variations at any $s_{h} \in \mathcal{H}_{h}$ is naturally identified with $H_{h}$.

In order to define the discrete energy functional $E_{h}$, analogous to Definition 3.3 of the energy functional $E$, we require the following discrete spaces.

\section{Definition 4.2.}

$$
\begin{aligned}
X_{h}^{n} & =\left\{u_{h} \in C^{0}\left(D_{h} ; \mathbb{R}^{n}\right) \mid u_{h} \in P_{1}(G) \text { for } G \in \mathcal{G}_{h}\right\}, \\
x_{h}^{n} & =\left\{f_{h} \in C^{0}\left(\partial D_{h} ; \mathbb{R}^{n}\right) \mid f_{h} \in P_{1}\left(E_{j}\right) \text { for } 1 \leq j \leq M\right\} .
\end{aligned}
$$

Taking $n=1$ we similarly define $X_{h}$ and $x_{h}$.

For $f_{h} \in x_{h}$ we define the discrete harmonic extension $\Phi_{h} f_{h} \in X_{h}$ by

$$
\begin{aligned}
\triangle_{h} \Phi_{h} f_{h} & =0 \text { in } D_{h}, \\
\Phi_{h} f_{h} & =f_{h} \text { on } \partial D_{h},
\end{aligned}
$$

where $\triangle_{h}$ is the discrete Laplacian. Thus (44) is interpreted in the weak sense:

$$
\int_{D_{h}} \nabla\left(\Phi_{h} f_{h}\right) \nabla \psi_{h}=0
$$

for all $\psi_{h} \in X_{h}$ such that $\psi_{h}=0$ on $\partial D_{h}$. If $f_{h} \in x_{h}^{n}$, then the discrete harmonic extension is defined componentwise.

\subsection{The discrete energy functional.}

Definition 4.3. For $s_{h} \in \mathcal{H}_{h}$, the discrete energy functional $E_{h}$ is defined by

$$
E_{h}\left(s_{h}\right)=\frac{1}{2} \int_{D_{h}}\left|\nabla \Phi_{h} I_{h}\left(\gamma \circ s_{h}\right)\right|^{2}=\mathcal{D}_{h}\left(\Phi_{h} I_{h}\left(\gamma \circ s_{h}\right)\right) .
$$

That is, $E_{h}\left(s_{h}\right)=\mathcal{D}_{h}\left(u_{h}\right)$, where $u_{h}$ is the discrete harmonic extension of $I_{h}\left(\gamma \circ s_{h}\right)$. We first apply $I_{h}$ to $\gamma \circ s_{h}$ since the latter is not in $x_{h}^{n}$, not being piecewise linear.

Note that, for a fixed parametrisation $\gamma, E_{h}\left(s_{h}\right)$ is completely determined by the nodal values $s_{h}\left(\phi_{j}\right)$, and can be computed from the values $\gamma \circ s_{h}\left(\phi_{j}\right)$ by solving a linear system of equations; see Section 5. In particular, if $\gamma \in C^{k}$, then $E_{h}\left(s_{h}\right)$ is also a $C^{k}$ function of $s_{h} \in \mathcal{H}_{h}$, as follows immediately from (60) since the $A_{i j}$ do not depend on $\gamma$. Finally, note that $E_{h}$ is of course not the restriction of $E$ to $\mathcal{H}_{h}$.

We are now ready to give the formulation of the discrete Plateau Problem which we use in this paper.

Definition 4.4. The discrete harmonic function

$$
u_{h}=\Phi_{h} I_{h}\left(\gamma \circ s_{h}\right)
$$

is a discrete minimal surface spanning $\Gamma$, or a solution of the discrete Plateau Problem for $\Gamma$, if and only if $s_{h} \in \mathcal{H}_{h}$ is stationary for $E_{h}$, i.e.,

$$
\left\langle E_{h}^{\prime}\left(s_{h}\right), \xi_{h}\right\rangle=0
$$

for all $\xi_{h} \in H_{h}$.

Note that we do not require monotonicity of $s_{h}$, as is the case for $s$ in Definition 3.6. 
For later use we compute from (47) that

$$
\begin{aligned}
E_{h}\left(s_{h}\right) & =\frac{1}{2} \int_{D_{h}}\left|\nabla u_{h}\right|^{2} \\
\left\langle E_{h}^{\prime}\left(s_{h}\right), \xi_{h}\right\rangle & =\left.\frac{d}{d t}\right|_{t=0} E_{h}\left(s_{h}+t \xi_{h}\right)=\int_{D_{h}} \nabla u_{h} \nabla v_{h}, \\
E_{h}^{\prime \prime}\left(s_{h}\right)\left(\xi_{h}, \xi_{h}\right) & =\left.\frac{d^{2}}{d t^{2}}\right|_{t=0} E_{h}\left(s_{h}+t \xi_{h}\right)=\int_{D_{h}} \nabla u_{h} \nabla w_{h}+\int_{D_{h}}\left|\nabla v_{h}\right|^{2},
\end{aligned}
$$

where

$$
u_{h}=\Phi_{h} I_{h}\left(\gamma \circ s_{h}\right), \quad v_{h}=\Phi_{h} I_{h}\left(\gamma^{\prime} \circ s_{h} \xi_{h}\right), \quad w_{h}=\Phi_{h} I_{h}\left(\gamma^{\prime \prime} \circ s_{h} \xi_{h}^{2}\right) .
$$

Bilinearity gives a corresponding expression for distinct variations in (51). The map $I_{h}\left(\gamma^{\prime} \circ s_{h} \xi_{h}\right)$ can be thought of as a discrete tangential vector field defined either along $\partial D_{h}$ or $\Gamma_{h}$ (the polygonal image of $\partial D_{h}$ under the map $I_{h}\left(\gamma \circ s_{h}\right)$ ); it is uniquely determined by its values $\gamma^{\prime}\left(s_{h}\left(\phi_{j}\right)\right) \xi_{h}\left(\phi_{j}\right)$ at boundary nodes $\phi_{j}$. The map $v_{h}$ is the discrete harmonic extension of $I_{h}\left(\gamma^{\prime} \circ s_{h} \xi_{h}\right)$ and can be thought of as the discrete harmonic variation of $u_{h}$ induced by $\xi_{h}$.

\section{The NUMERICAL ALGORITHM}

We now describe our algorithm for the computation of discrete minimal surfaces. We want to solve the equation

$$
E_{h}^{\prime}\left(s_{h}\right)=0
$$

in the discrete space $\mathcal{H}_{h}$. This is equivalent to computing $s_{h}$ such that

$$
\left\langle E_{h}^{\prime}\left(s_{h}\right), \xi_{h}\right\rangle=0 \quad \forall \xi_{h} \in H_{h} .
$$

The abstract Newton algorithm for the solution is the following.

Algorithm 5.1. Given an initial parametrization $s_{h} \in \mathcal{H}_{h}$ and a tolerance $\epsilon>0$ :

1. Compute $E_{h}^{\prime}\left(s_{h}\right)$.

2. If $\left\|E_{h}^{\prime}\left(s_{h}\right)\right\|_{H_{h}{ }^{\prime}} \leq \epsilon$, then go to step 5 .

3. Solve the linear problem

$$
E_{h}^{\prime \prime}\left(s_{h}\right)\left(\eta_{h}, \xi_{h}\right)=-\left\langle E_{h}^{\prime}\left(s_{h}\right), \xi_{h}\right\rangle \quad \forall \xi_{h} \in H_{h} .
$$

4. Update the solution: $s_{h}:=s_{h}+\eta_{h}$ and go to step 1 .

5. Compute the discrete minimal surface

$$
u_{h}=\Phi_{h} I_{h}\left(\gamma \circ s_{h}\right)
$$

and stop.

In order to efficiently implement this algorithm we will translate it into matrixvector form. Recall that $M$ is the number of boundary nodes on $\partial D$ and $N$ is the number of nodes in $\bar{D}_{h}$. Recall $X_{h}$ is the space defined in (42) with $n=1$. By

$$
\bar{H}_{h}\left(\supset H_{h}\right)
$$

we mean the space defined as is $H_{h}$, but without the normalisation condition (21). The nodal basis functions $\zeta_{h}^{(i)} \in \bar{H}_{h}$ and $\psi_{h}^{(l)} \in X_{h}$ are uniquely specified by

$$
\zeta_{h}^{(i)}\left(\phi_{j}\right)=\delta_{i j} \quad \text { and } \quad \psi_{h}^{(l)}\left(x_{m}\right)=\delta_{l m},
$$

where $i, j=1, \ldots, M$ and $l, m=1, \ldots, N$. 
For the remainder of this section, subscripts $i, j, k$ will range from 1 to $M$, and subscripts $l, m$ will range from 1 to $N$.

For $s_{h} \in \mathcal{H}_{h}, \xi_{h} \in \bar{H}_{h}$ and $u_{h} \in X_{h}^{n}$ let

$$
s_{i}=s_{h}\left(\phi_{i}\right) \in S^{1}, \quad \xi_{i}=\xi_{h}\left(\phi_{i}\right) \in \mathbb{R}, \quad u_{l}=u_{h}\left(x_{l}\right) \in \mathbb{R}^{n} .
$$

Then

$$
\xi_{h}=\sum_{i=1}^{M} \xi_{i} \zeta_{h}^{(i)}, \quad u_{h}=\sum_{l=1}^{N} u_{l} \psi_{h}^{(l)}
$$

The coefficient vectors will be written

$$
\begin{gathered}
\boldsymbol{s}=\left(s_{1}, \ldots, s_{M}\right) \in\left(S^{1}\right)^{M}, \quad \boldsymbol{\xi}=\left(\xi_{1}, \ldots, \xi_{M}\right) \in \mathbb{R}^{M} \\
\boldsymbol{u}=\left(u_{1}, \ldots, u_{N}\right) \in\left(\mathbb{R}^{n}\right)^{N} .
\end{gathered}
$$

Given $\boldsymbol{\xi} \in \mathbb{R}^{M}$ the corresponding function $\xi_{h}$ belongs to $H_{h}$ iff the normalisations corresponding to (21) are satisfied. It is easily seen that this is equivalent to

$$
\begin{aligned}
\sum_{i=1}^{M} \frac{\xi_{i+1}+\xi_{i}}{2}\left(\phi_{i+1}-\phi_{i}\right) & =0 \\
\sum_{i=1}^{M} \frac{\xi_{i+1}-\xi_{i}}{\phi_{i+1}-\phi_{i}}\left(\cos \phi_{i+1}-\cos \phi_{i}\right) & =0 \\
\sum_{i=1}^{M} \frac{\xi_{i+1}-\xi_{i}}{\phi_{i+1}-\phi_{i}}\left(\sin \phi_{i+1}-\sin \phi_{i}\right) & =0
\end{aligned}
$$

where $\phi_{M+1}=\phi_{1}+2 \pi$. We write the above linear constraints as

$$
\boldsymbol{L} \boldsymbol{\xi}=\mathbf{o}
$$

We will need to compute $E_{h}^{\prime}\left(s_{h}\right)$ and $E_{h}^{\prime \prime}\left(s_{h}\right)$; see (50) and (51). For this, first note that from the definition of $I_{h}$ we have

$$
\begin{aligned}
I_{h}\left(\gamma \circ s_{h}\right) & =\left.\sum_{i=1}^{M} \psi_{h}^{(i)}\right|_{\partial D_{h}} \gamma\left(s_{i}\right), \\
I_{h}\left(\gamma^{\prime} \circ s_{h} \xi_{h}\right) & =\left.\sum_{i=1}^{M} \xi_{i} \psi_{h}^{(i)}\right|_{\partial D_{h}} \gamma^{\prime}\left(s_{i}\right), \\
I_{h}\left(\gamma^{\prime \prime} \circ s_{h} \xi_{h} \eta_{h}\right) & =\left.\sum_{i=1}^{M} \xi_{i} \eta_{i} \psi_{h}^{(i)}\right|_{\partial D_{h}} \gamma^{\prime \prime}\left(s_{i}\right) .
\end{aligned}
$$

In order to compute the effect of the discrete harmonic operator $\Phi_{h}$ applied to the above quantities, we need the (scaled) discrete Poisson kernel functions. More precisely, for $i=1, \ldots, M$, the functions $K_{h}^{(i)}=\Phi_{h}\left(\left.\psi_{h}^{(i)}\right|_{\partial D_{h}}\right) \in X_{h}$ are uniquely defined by

$$
\begin{aligned}
\int_{D_{h}} \nabla K_{h}^{(i)} \nabla \psi_{h}^{(l)} & =0 \quad \text { for } M<l \leq N \\
K_{h}^{(i)} & =\psi_{h}^{(i)} \quad \text { on } \partial D_{h}
\end{aligned}
$$

see $(44)-(46)$. 
In order to compute the $K_{h}^{(i)}$ and for later use, define the $N \times N$ stiffness matrix $\boldsymbol{S}=\left[S_{l m}\right]$ by

$$
S_{l m}=\int_{D_{h}} \nabla \psi_{h}^{(l)} \nabla \psi_{h}^{(m)}
$$

The $N \times N$ matrix $\boldsymbol{S}^{0}=\left[S_{l m}^{0}\right]$, and the $N$-vectors $\boldsymbol{b}^{(i)}=\left(b_{l}^{(i)}\right)$ for $i=1, \ldots, M$, are defined by

$$
S_{l m}^{0}=\left\{\begin{array}{ll}
\delta_{l m} & 1 \leq l, m \leq M \\
S_{l m} & M<l, m \leq N \\
0 & \text { otherwise }
\end{array} \quad \text { and } \quad b_{l}^{(i)}= \begin{cases}\delta_{i l} & 1 \leq l \leq M \\
-S_{i l} & M<l \leq N .\end{cases}\right.
$$

Note that $\boldsymbol{S}^{0}$ is a symmetric matrix with 4 blocks, of which the upper right and lower left are zero matrices.

Let

$$
K_{h}^{(i)}=\sum_{l=1}^{N} K_{l}^{(i)} \psi_{h}^{(l)}
$$

Then from (54) and (55), the $N$-vectors

$$
\boldsymbol{K}^{(i)}=\left(K_{1}^{(i)}, \ldots, K_{N}^{(i)}\right)
$$

for $i=1, \ldots, M$ are each given by solving the system of equations

$$
\boldsymbol{S}^{(0)} \boldsymbol{K}^{(i)}=\boldsymbol{b}^{(i)} \text {. }
$$

Note that the matrix $\boldsymbol{S}^{(0)}$ is independent of $i$.

The $M \times M$ harmonic stiffness matrix $\boldsymbol{A}$ is defined by

$$
A_{i j}=\int_{D_{h}} \nabla K_{h}^{(i)} \nabla K_{h}^{(j)}=\int_{D_{h}} \nabla K_{h}^{(i)} \nabla \psi_{h}^{(j)},
$$

and from the preceding we have

$$
A_{i j}=\sum_{l, m=1}^{N} K_{l}^{(i)} K_{m}^{(j)} S_{l m}=\sum_{l=1}^{N} K_{l}^{(i)} S_{l j}
$$

Of course, for computational purposes the second expression is much more efficient.

In terms now of previously computed quantities, it follows from (47), (50), (51) and the preceding that

$$
\begin{aligned}
E_{h}\left(s_{h}\right) & =\frac{1}{2} \sum_{i, j=1}^{M} A_{i j} \gamma\left(s_{i}\right) \cdot \gamma\left(s_{j}\right), \\
\text { (61) }\left\langle E_{h}^{\prime}\left(s_{h}\right), \xi_{h}\right\rangle & =\sum_{i, j=1}^{M} A_{i j} \gamma\left(s_{i}\right) \cdot \gamma^{\prime}\left(s_{j}\right) \xi_{j}, \\
(62) E_{h}^{\prime \prime}\left(s_{h}\right)\left(\eta_{h}, \xi_{h}\right) & =\sum_{i, j=1}^{M} A_{i j}\left(\gamma\left(s_{i}\right) \cdot \gamma^{\prime \prime}\left(s_{j}\right) \xi_{j} \eta_{j}+\gamma^{\prime}\left(s_{i}\right) \cdot \gamma^{\prime}\left(s_{j}\right) \xi_{i} \eta_{j}\right) .
\end{aligned}
$$

Moreover, the discrete harmonic surface corresponding to $s_{h}$ is given by

$$
u_{h}=\Phi_{h} I_{h}\left(\gamma \circ s_{h}\right)=\sum_{i=1}^{M} K_{h}^{(i)} \gamma\left(s_{i}\right)=\sum_{l=1}^{N}\left(\sum_{i=1}^{M} K_{l}^{(i)} \gamma\left(s_{i}\right)\right) \psi_{h}^{(l)} .
$$


We now give the previous algorithm in matrix-vector form. In the following, the vector $\boldsymbol{g}$ and the matrix $\boldsymbol{B}$ correspond to $E_{h}^{\prime}\left(s_{h}\right)$ and $E_{h}^{\prime \prime}\left(s_{h}\right)$, respectively, while the equation $E_{h}^{\prime \prime}\left(s_{h}\right)\left(\eta_{h}, \xi_{h}\right)=-\left\langle E_{h}^{\prime}\left(s_{h}\right), \xi_{h}\right\rangle \quad \forall \xi_{h} \in H_{h}$ becomes the equation $\boldsymbol{B} \boldsymbol{\eta}=-\boldsymbol{g}$ together with the (constraint) equation $\boldsymbol{L} \boldsymbol{\eta}=\mathbf{o}$.

Algorithm 5.2. Suppose a triangulation $D_{h}$ of the unit disc is given:

1. Compute the $N \times N$ stiffness matrix $\boldsymbol{S}$,

$$
\boldsymbol{S}=S_{l m}=\int_{D_{h}} \nabla \psi_{h}^{(l)} \nabla \psi_{h}^{(m)}
$$

2. Define $\boldsymbol{S}^{0}$ and $\boldsymbol{b}^{(i)}$ as in (57) and solve the $N \times N$ systems of equations

$$
\boldsymbol{S}^{0} \boldsymbol{K}^{(i)}=\boldsymbol{b}^{(i)}
$$

for $i=1, \ldots, M$.

3. Compute and save the $M \times M$ harmonic stiffness matrix

$$
\boldsymbol{A}=A_{i j}=\sum_{l=1}^{N} K_{l}^{(i)} S_{l j}
$$

Suppose, furthermore, a parametrised curve $\gamma$, an initial vector $s_{h} \in \mathcal{H}_{h}$, and a tolerance $\epsilon>0$ are given. Then:

4. Compute the $M$-dimensional "gradient vector" $\boldsymbol{g}=\left(g_{j}\right)$,

$$
g_{j}=\sum_{i=1}^{M} A_{i j} \gamma\left(s_{i}\right) \cdot \gamma^{\prime}\left(s_{j}\right)
$$

5. If $|\boldsymbol{g}| \leq \epsilon$, then go to step 8 .

6. Solve the $M \times M$ linear system of equations

$$
\boldsymbol{B} \boldsymbol{\eta}=-\boldsymbol{g}, \quad \boldsymbol{L} \boldsymbol{\eta}=\mathbf{o}
$$

where $\boldsymbol{B}=\left[B_{i j}\right]$ is the $M \times M$ matrix

$$
B_{i j}=A_{i j} \gamma^{\prime}\left(s_{i}\right) \cdot \gamma^{\prime}\left(s_{j}\right)+\delta_{i j} \sum_{k=1}^{M} A_{k j} \gamma\left(s_{k}\right) \cdot \gamma^{\prime \prime}\left(s_{j}\right),
$$

and $\boldsymbol{L}$ is as in (53).

7. Update the solution

$$
s:=s+\eta
$$

and go to step 4.

8. Compute the discrete minimal surface

$$
u_{h}=\Phi_{h} I_{h}\left(\gamma \circ s_{h}\right)=\sum_{l=1}^{N} u_{l} \psi_{h}^{(l)}
$$

by solving

$$
\boldsymbol{S}^{(0)} \boldsymbol{u}=\gamma
$$

where

$$
\begin{aligned}
\boldsymbol{u} & =\left(u_{1}, \ldots, u_{M}, \ldots, u_{N}\right) \\
\boldsymbol{\gamma} & =\left(\gamma\left(s_{1}\right), \ldots, \gamma\left(s_{M}\right), 0, \ldots, 0\right)
\end{aligned}
$$

and stop. 
Note that the computation of the harmonic stiffness matrix $\boldsymbol{A}$ depends only on the triangulation, and not on $\gamma$ or $s_{h}$. Thus steps 1-3 may be performed just once independently of $\gamma$ for each grid; in steps $4-7$ only $\boldsymbol{A}$ is needed from steps $1-3$. The sparse matrix $\boldsymbol{S}$ is used in step 8. It is not necessary to store the $\boldsymbol{K}^{(i)}$. In particular, it is better to solve the system in step 8 directly, rather than saving the $\boldsymbol{K}^{(i)}$ and using (63) to find $\boldsymbol{u}$.

The algorithm requires $O\left(N^{3 / 2}\right)$ operations. Each of the $M$ sparse equations in step 2 can typically be solved in $O(N)$ operations to within a prescribed error by a conjugate gradient algorithm, and $M=O\left(N^{1 / 2}\right)$. Step 3 requires $O\left(M^{2}\right)=$ $O(N)$ operations, because of the sparsity of $\boldsymbol{S}$. Thus the initial set-up requires $O\left(N^{3 / 2}\right)$ operations. Step 4 requires $O\left(M^{2}\right)=O(N)$ operations. In step 6 the computation of $\boldsymbol{B}$ requires $O\left(M^{2}\right)=O(N)$ operations, and the solution of the (full) system requires $O\left(M^{2}\right)=O(N)$ operations by an iterative method. Thus each loop through steps $4-7$ requires $O(N)$ operations. The number of loops required to find the $M$ entries in $\boldsymbol{g}$ is typically $O(M)$. Finally, the sparse system in step 8 can be solved in $O(N)$ operations by a conjugate gradient method.

\section{ImPlementation AND NUMERICAL RESUlTS}

The classical Enneper surface with parameter $R$, which acts as a bifurcation parameter, is a good test example because here the exact solution for the unstable case is known. For $0<R \leq 1$ Enneper's surface is area minimising and is the unique solution of Plateau's Problem. For $1<R<\sqrt{3}$ one observes three distinct solutions of Plateau's Problem - two area minimisers and one unstable minimal surface, but this has only been proved if $r_{0}<R<\sqrt{3}$ for some $r_{0}$ near $\sqrt{3}$. For a discussion of this important example and further references, see [N2], particularly $\S \S 118,388-395$, A14-16. Here, we compute the unstable solution and calculate the order of convergence between the smooth solution and the discrete solution.

The boundary curve is given by

$$
\begin{aligned}
& \gamma^{1}(s)=R \cos s-R^{3} / 3 \cos 3 s \\
& \gamma^{2}(s)=R \sin s+R^{3} / 3 \sin 3 s \\
& \gamma^{3}(s)=R^{2} \cos 2 s
\end{aligned}
$$

for $s \in[0,2 \pi]$. The continuous solution is the harmonic continuation of this parametrisation. Denote by $e_{h}$ the error between the continuous solution and the discrete solution. For two successive grids with grid sizes $h_{1}$ and $h_{2}$ the experimental order of convergence is

$$
e o c=\ln \frac{e_{h_{1}}}{e_{h_{2}}} / \ln \frac{h_{1}}{h_{2}} .
$$

In each of the three cases a different grid was used for the computations in order to make the comparison of the orders of convergence more realistic. The grids are based on initial macro triangulations of the unit disk which are of quadrilateral, hexagonal, or pentagonal form. 


\begin{tabular}{|r|l|l|l|l|l|l|}
\hline \multicolumn{7}{|c|}{ Stable Enneper Surface, $\mathbf{R}=\mathbf{0 . 9}$, hexagonal grid } \\
\hline level & $h$ & $L^{2}$-error & eoc $L^{2}$ & $H^{1}$-error & eoc $H^{1}$ & energy $E$ \\
\hline \hline 0 & 1.0000 & 1.9557 e- 1 & - & 1.3301 & - & 3.8318 \\
\hline 1 & 0.5176 & 1.0617 e-1 & 0.928 & 8.5964 e- 1 & 0.663 & 4.8395 \\
\hline 2 & 0.3098 & 3.1492 e- 2 & 2.368 & 4.4964 e- 1 & 1.263 & 5.0842 \\
\hline 3 & 0.1685 & 7.8183 e-3 & 2.288 & 2.2709 e- 1 & 1.122 & 5.1432 \\
\hline 4 & 0.0875 & 1.9786 e-3 & 2.095 & 1.1389 e- 1 & 1.052 & 5.1576 \\
\hline 5 & 0.0445 & 3.1723 e-4 & 2.709 & $5.6979 \mathrm{e}-2$ & 1.025 & 5.1612 \\
\hline
\end{tabular}

\begin{tabular}{|r|l|l|l|l|l|l|}
\hline \multicolumn{8}{|c|}{ Enneper Surface, R $=1.0$, pentagonal grid } \\
\hline level & $h$ & $L^{2}$-error & eoc $L^{2}$ & $H^{1}$-error & eoc $H^{1}$ & energy $E$ \\
\hline \hline 1 & 0.6641 & 1.0586 e- 1 & - & 1.1595 & - & 6.5176 \\
\hline 2 & 0.3320 & 2.9051 e- 2 & 1.866 & 6.3118 e- 1 & 0.877 & 7.1272 \\
\hline 3 & 0.1843 & 7.4972 e- 3 & 2.302 & 3.2300 e- 1 & 1.138 & 7.2799 \\
\hline 4 & 0.0964 & 1.8916 e- 3 & 2.124 & 1.6250 e- 1 & 1.060 & 7.3178 \\
\hline 5 & 0.0492 & 4.7398 e- 4 & 2.058 & 8.1384 e- 2 & 1.028 & 7.3272 \\
\hline
\end{tabular}

\begin{tabular}{|r|l|l|l|l|l|l|}
\hline \multicolumn{6}{|c|}{ Unstable Enneper Surface, R=1.1, quadrilateral grid } \\
\hline level & $h$ & $L^{2}$-error & eoc $L^{2}$ & $H^{1}$-error & eoc $H^{1}$ & energy $E$ \\
\hline \hline 1 & 0.7654 & 1.8741 e- 1 & - & 1.5713 & - & 8.1395 \\
\hline 2 & 0.3902 & 4.9817 e- 2 & 1.967 & 8.8969 e- 1 & 0.844 & 9.6962 \\
\hline 3 & 0.2102 & 1.2713 e- 2 & 2.207 & 4.6046 e- 1 & 1.065 & 10.1150 \\
\hline 4 & 0.1110 & 3.1975 e- 3 & 2.161 & 2.3238 e-1 & 1.071 & 10.2208 \\
\hline 5 & 0.0569 & 8.0072 e- 4 & 2.071 & 1.1647 e- 1 & 1.033 & 10.2473 \\
\hline
\end{tabular}

In order to demonstrate the sharpness of our error estimates we include an example from [DH3]. We do not prove convergence if the kernel of the second derivatives of $E$ is nontrivial. The following experiment shows that in general these results cannot be improved. The example uses the exact formula for a minimal surface $u=\Phi(\gamma \circ s)$ with a branch point at the origin. In this case the kernel of $E^{\prime \prime}(s)$ is well known and we are able to subtract the singular part of the solution, i.e., to project the solution onto the space orthogonal to the kernel and so obtain the regular part.

\begin{tabular}{|r|l|l|l|l|l|}
\hline \multicolumn{7}{|c|}{ Branch point (order=1, index=3) } \\
\hline level & $h$ & $L^{2}$-error & eoc $L^{2}$ & $H^{1}$-error & eoc $H^{1}$ \\
\hline \hline 2 & 0.3902 & 7.151 e- 3 & - & 3.455 e- 2 & - \\
\hline 3 & 0.2102 & 7.817 e- 3 & -0.1 & 2.875 e- 2 & 0.3 \\
\hline 4 & 0.1110 & 1.119 e- 2 & -0.6 & 3.920 e- 2 & -0.5 \\
\hline
\end{tabular}




\begin{tabular}{|r|l|l|l|l|l|}
\hline \multicolumn{7}{|c|}{ Branch point: regular part } \\
\hline level & $h$ & $L^{2}$-error & eoc $L^{2}$ & $H^{1}$-error & eoc $H^{1}$ \\
\hline \hline 2 & 0.3902 & $3.761 \mathrm{e}-3$ & - & $1.878 \mathrm{e}-2$ & - \\
\hline 3 & 0.2102 & $7.292 \mathrm{e}-4$ & 2.7 & $3.879 \mathrm{e}-3$ & 2.6 \\
\hline 4 & 0.1110 & $9.881 \mathrm{e}-5$ & 3.1 & $5.977 \mathrm{e}-4$ & 2.9 \\
\hline
\end{tabular}

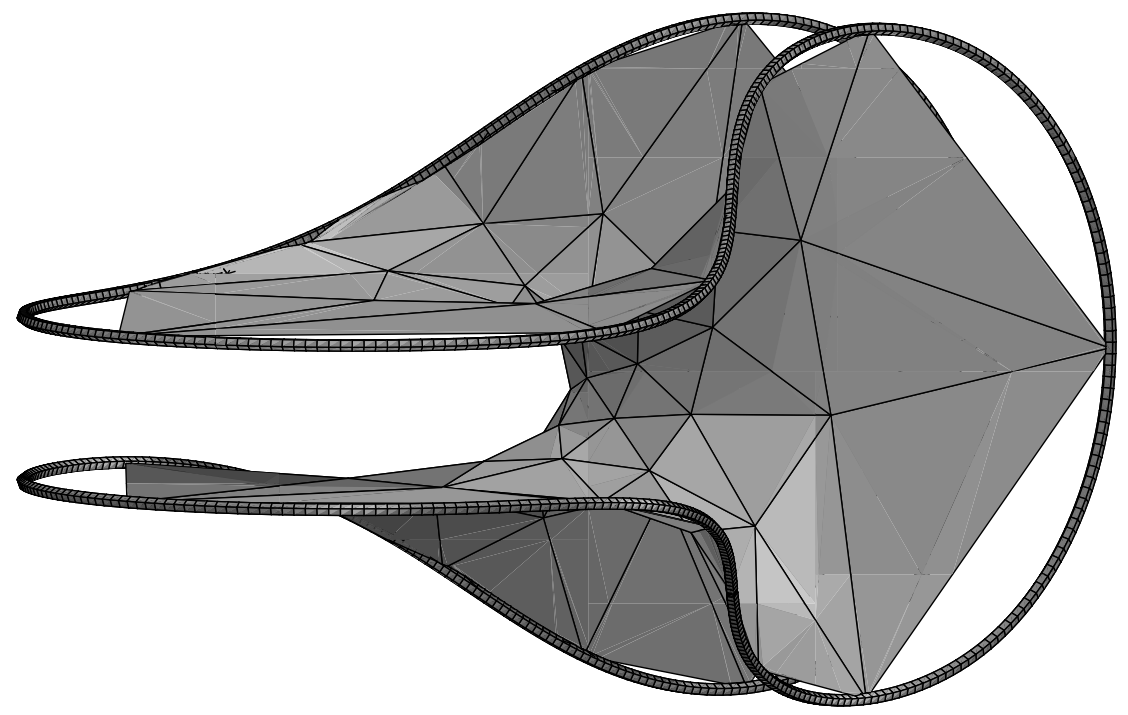

Figure 2. Discrete solution of the Plateau Problem with 96 triangles

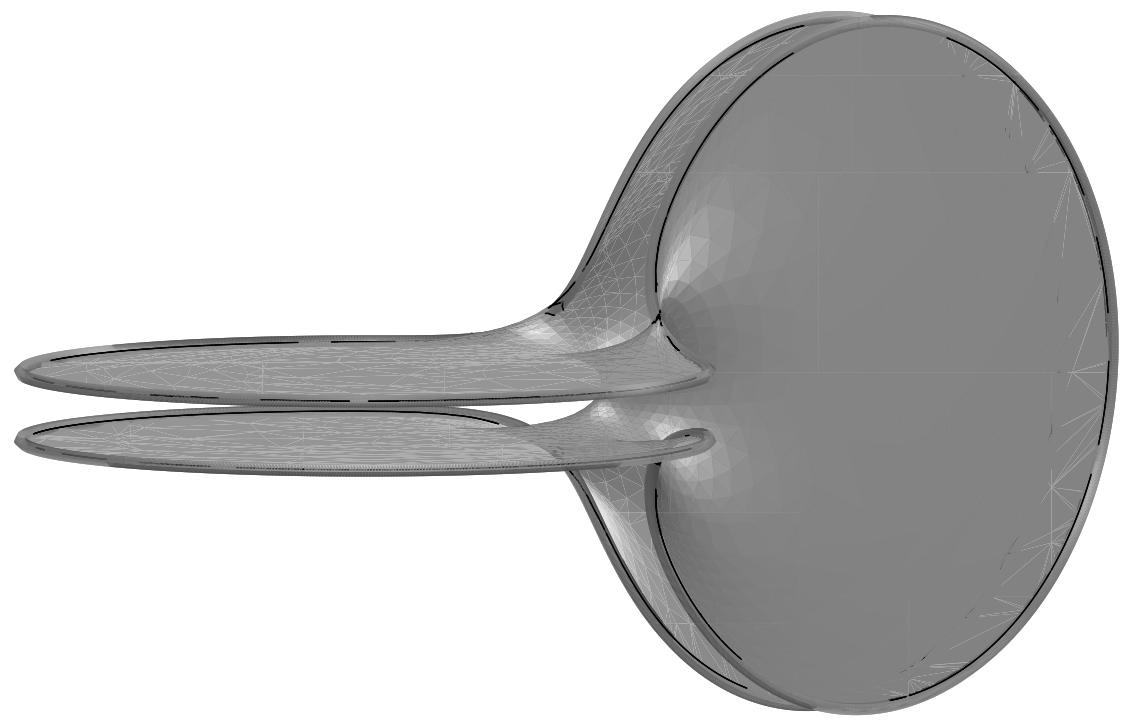

Figure 3. Discrete solution of the Plateau Problem with 1536 triangles 
Finally we compute a nonminimising solution for a well-known boundary curve consisting of four circles, parallel in pairs and connected in a smooth way. The distance between two parallel circles is $\epsilon=0.5$ for the coarse grid in Figure 2 and $\epsilon=0.2$ for the fine grid in Figure 3. The radii of the circles are 1.0 in either case. The four connections between the circles are of length proportional to $\epsilon$ and thus at these parts of the boundary very high curvatures occur. These curvatures are equivalent to large $C^{2}$-norms of the parametrisation $\gamma$ and, as can be seen from the constants in our error estimates, large errors between discrete and continuous

solution can be expected at these points. In fact, these parts of the discrete minimal surface induce many Newton steps in our algorithm.

\section{REFERENCES}

[A1] H.W. Alt, Verzweigungspunkte von H-Flächen I, Math. Zeit. 127 (1972), 333-362. MR 47:965b

[A2] H.W. Alt, Verzweigungspunkte von H-Flächen II, Math. Ann. 201 (1973), 33-55. MR 48:9529

[Br] K.A. Brakke, The Surface Evolver, Exp. Math. 1 (1992), 141-165. MR 93k:53006

[BT] R. Böhme \& T. Tromba, The Index Theorem for Classical Minimal Surfaces, Ann. Math. 113 (1981), 447-499. MR 83a:58031

[Ci] P.G. Ciarlet, The Finite Element Methods for Elliptic Problems, North Holland 1978. MR 58:25001

[Co] P. Concus, Numerical Solution of the Minimal Surface Equation, Math. Comp. 21 (1967), 340-350. MR 37:4968

[Cou] R. Courant, Dirichlet's Principle, Conformal Mapping and Minimal Surfaces, Interscience 1950. MR 12:90a

[Dou] J. Douglas, Solution of the Problem of Plateau, Trans. Am. Math. Soc. 33 (1931), 263-321.

[Dou2] J. Douglas, A Method of Numerical Solution of the Plateau Problem, Ann. Math. (2) 29 (1928), 180-188.

[Dz] G. Dziuk, An Algorithm for Evolutionary Surfaces, Num. Math. 58 (1991), 603-611. MR 91k:65042

[DH0] G. Dziuk, J.E. Hutchinson, On the approximation of unstable parametric minimal surfaces, preprint No. 340 (1994) SFB 256, Bonn, or CMA Math. Res. Rep. 9 (1994), Australian National University.

[DH1] G. Dziuk, J.E. Hutchinson, On the approximation of unstable parametric minimal surfaces, Calc. Var. 4 (1996), 27-58. MR 96m:49073

[DH2] G. Dziuk, J.E. Hutchinson, $L^{2}$ Estimates for Approximations to Minimal Surfaces, Proceedings of the International Conference: Curvature Flows and Related Topics, 67-92, A. Damlamian, J. Spruck \& A. Visintin eds., Gakkotosho, Tokyo, 1995. MR 97g:58042

[DH3] G. Dziuk, J.E. Hutchinson, A Finite Element Method for the Computation of Parametric Minimal Surfaces, Tatra Mount. Math. J. 4 (1994), 49-62. MR 95g:65151

[DH4] G. Dziuk, J.E. Hutchinson, The Discrete Plateau Problem: Convergence Results, to appear.

[DHKW] U. Dierkes, S. Hildebrandt, A. Küster \& O. Wohlrab, Minimal Surfaces I \& II, Grundlehren der Mathematischen Wissenschaften 295-6, Springer-Verlag 1992. MR 94c:49001a; MR 94c:49001b

[G] R. Gulliver, Regularity of Minimizing Surfaces of Prescribed Mean Curvature, Ann. Math. 97 (1973), 275-305. MR 47:5736

[He] E. Heinz, Über das Randverhalten quasilinearer elliptischer Systeme mit isothermen Parametern, Math. Zeit. 113 (1970), 99-105. MR 41:7288

[Hi1] M. Hinze, On the Numerical Treatment of Quasiminimal Surfaces, Preprint 315 TU Berlin 1992. MR 95b:49063

[Hi2] M. Hinze, On a Simple Method to Compute Polygonal Minimal Surfaces, Preprint 33 SFB 288, Berlin 1992. 
[Hil] S. Hildebrandt, Boundary Behaviour of Minimal Surfaces, Arch. Rat. Mech. Anal. 35 (1969), 47-82. MR 40:1901

[Hu] J.E. Hutchinson, Computing Conformal Maps and Minimal Surfaces, Proc. C.M.A., Canberra 26 (1991), 140-161. MR 92m:53012

[Ja] W. Jäger, Das Randverhalten von Flächen beschränkter mittlerer Krümmung bei $C^{1, \alpha}$ Rändern, Nachr. Akad. Wiss. Gött., II. Math. Phys. Kl. (1977), Nr. 5. MR 58:29204

[J] H. Jarausch, Zur numerischen Behandlung von parametrischen Minimalflächen mit Finite-Elementen, Dissertation Bochum 1978.

[JT] C. Johnson \& V. Thomée, Error Estimates for a Finite Element Approximation of a Minimal Surface, Math. Comp. 29 (1975), 343-349. MR 53:4571

[N1] J.C.C. Nitsche, The Boundary Behaviour of Minimal Surfaces. Kellogg's Theorem and Branch Points on the Boundary, Invent. Math. 8 (1969), 313-333. MR 41:4399a

[N2] J.C.C. Nitsche, Lectures on Minimal Surfaces Volume 1, Cambridge University Press 1989. MR 90m:49031

[O] R. Osserman, A Proof of the Regularity Everywhere of the Classical Solution to Plateau's Problem, Ann. Math. 91 (1970), 550-569. MR 42:979

[P] H. Parks, Explicit Determination of Area Minimizing Hypersurfaces II, Mem. A.M.S. 60 (1986), no. 342 . MR 87h:49045

[PP] U. Pinkall, K. Polthier, Computing Discrete Minimal Surfaces and Their Conjugates, Exp. Math. 2 (1993), 15-36. MR 94j:53009

[R] T. Rado, On Plateau's Problem, Ann. Math. 2 (1930), 457-469.

[Ra] R. Rannacher, Some Asymptotic Error Estimates for Finite Element Approximation of a Minimal Surface, Rev. Française Automat. Informat. Recherche Opérationnelle Sér Rouge Anal. Numér. 11 (1977), 181-196. MR 56:4199

[St1] M. Struwe, On a Critical Point Theory for Minimal Surfaces Spanning a Wire, J. Reine Angew. Math 349 (1984), 1-23. MR 87a:58045

[St2] M. Struwe, Plateau's Problem and the Calculus of Variations, Mathematical Notes 35, Princeton University Press 1988. MR 90h:58016

[Ste] G. Steinmetz, Numerische Approximation von allgemeinen parametrischen Minimalflächen im $\mathbb{R}^{3}$, Forschungsarbeit FHS Regensburg 1987.

[Su] J. Sullivan, A Crystalline Approximation Theorem for Hypersurfaces, Princeton Ph.D. thesis 1990.

[To] F. Tomi, On the finite solvability of Plateau's problem, Lect. Notes Math. 597, Springer, 679-695, 1977. MR 56:13118

[T1] T. Tsuchiya, On Two Methods for Approximation Minimal Surfaces in Parametric Form, Math. Comp. 46 (1986), 517-529. MR 87d:49043

[T2] T. Tsuchiya, Discrete Solution of the Plateau Problem and its Convergence, Math. Comp. 49 (1987), 157-165. MR 88i:49032

[T3] T. Tsuchiya, A Note on Discrete Solutions of the Plateau Problem, Math. Comp. 54 (1990), 131-138. MR 91c:49063

[U] A. Underwood, Constructing Barriers to Minimal Surfaces from Polyhedral Data, Princeton Ph.D. thesis 1993.

[Wa1] H. J. Wagner, Ein Beitrag zur numerischen Approximation von Minimalflächen, Computing 19 (1977), 35-58.

[Wa2] H. J. Wagner, Consideration of Obstacles in the Numerical Approximation of Minimal Surfaces, Computing 19/4 (1978), 375-378. MR 58:3567

[Wi] W. L. Wilson jr., On Discrete Dirichlet and Plateau Problem, Num. Math. 3 (1961), 359-373. MR 25:761

[Wo] O. Wohlrab, Zur numerischen Behandlung von parametrischen Minimalflächen mit halbfreien Rändern, Dissertation Bonn 1985.

Institut für Angewandte Mathematik, Universität Freiburg, Hermann-Herder-Str. 10, D-79104 Freiburg I. BR., GERMANY

E-mail address: gerd@mathematik.uni-freiburg.de

Department of Mathematics, School of Mathematical Sciences, Australian National University, GPO Box 4, Canberra, ACT 0200, AUSTRAliA

E-mail address: John.Hutchinson@anu.edu.au 